\title{
Facebook Translation Service (FTS) Usage among Jordanians during COVID-19 Lockdown
}

\author{
Zakaryia Almahasees ${ }^{1, *}$, Helene Jaccomard ${ }^{2}$ \\ ${ }^{1}$ Applied Science Private University, Department of English Language and Translation, Faculty of Arts and Science, Amman, 00962, \\ Jordan \\ ${ }^{2}$ University of Western Australia, Department of Translation, School of Humanities, Perth, 0061, Australia
}

\begin{tabular}{l} 
A R T I C L E I N F O \\
\hline Article history: \\
Received: 04 September, 2020 \\
Accepted: 30 October, 2020 \\
Online: 20 November, 2020
\end{tabular}

Keywords:

Facebook Translation service

Facebook and Covid-19

Facebook Posts

Translation Studies

English and Arabic

\begin{abstract}
A B S T R A C T
The world is facing an unprecedented virus outbreak, COVID-19, hitting more than 200 countries. Governments have been striving to prevent the spread of the virus through the lockdown. During the strict lockdown in Jordan, people needed to stay home, and they used available social networks to keep updated on COVID-19, with Facebook, the most popular social media platform. The study aimed to elicit information about assessing the use of FTS as a source of information in general and on COVID-19, in particular, FTS for those interested in English posts. However, it cannot read them and how reliable users think FTS is. The questionnaire was sent through the available networking sites, such as Facebook Messenger. The study found that 94.3\% use Facebook daily; $87.1 \%$ of the participants activated Facebook Translation Service (FTS). It is found that $62.2 \%$ of the participants considered Facebook as a primary source of information regarding COVID-19 and 27.8\% as secondary source. In terms of FTS usage, 87.3\% used FTS in translating English Facebook posts into Arabic, and 83.8\% used FTS in translating English Facebook COVID19 posts into Arabic during the lockdown. On the other hand, it is found that the majority found FTS committed minor errors in terms of adequacy and fluency. This success is due to the usage of Neural Machine Translation (NMT)approach and bilingual text corpora. The advantage is that FTS is a well-trained database that can provide more accurate translation than other model. In conclusion, disregarding FTS output quality, our research shows that Facebook and FTS became a significant source of information during abrupt crises. Such research would encourage government officials to better use Facebook and FTS as complements to their national health campaigns.
\end{abstract}

\section{Introduction}

The translation is the medium of human communications. It bridges the gap between human communities and ensures the best communication among the global communities [1]. Nowadays, the world is facing the most frightening Coronavirus (COVID-19) outbreak in the last century. International Health Organizations, Medical Specialists, health authorities, political leaders, and the public write posts round the clock on social networks, making the influx of information massive. The flow of information is unprecedented, and therefore, there should be a way to facilitate transferring this information into all-natural languages. It is predicted that the demand for translation industry will reach unprecedented number $\$ 56$ billion in 2021 [2]. This growth in

"Corresponding Author: Zakaryia Almahasees, Email: z_almhasees@asu.edu.jo www.astesi.com

https://dx.doi.org/10.25046/aj050661 translation demand has been occurring at a rate beyond professional human translators' capacity to keep up with, and the cost associated with human translation is too high for many purposes [3]. For example, It is indicated that they can translate up to 10 billion words daily. Translating the massive flow of information is beyond human translators' capacity, hence the central role of Machine Translation (MT). This tool can cope with large amounts of data at a split-second rate. However, there is no relation between the speed of translation and the quality of the translation [4].

In facing COVID-19, MT works as a tool to challenge and fight the virus. It is stated that "The challenge is how to communicate rapidly changing data across language borders, so that essential information is not lost in translation." In this concern, it has been shown that machine translation can help fight COVID-19. The 
current study aims to assess the popularity and use of Facebook Translation Service (FTS) in translating English Facebook posts in general and COVID-19 in particular among Facebookers in Jordan.

Social Networking is a flagship of human communications nowadays. Social networking refers to online platforms to keep humans connected regardless of their locations and territories. Social networking connects people globally of different groups and ethnicities for social, business and other purposes, such as Facebook, Twitter and the like [5]. More than 3.81 billion users of social networks worldwide and lists the most famous six social networking sites worldwide based on their monthly users: Facebook, YouTube, WhatsApp, Facebook Messenger, Weixin(WeChat), and then Instagram. Facebook tops the other social networking sites with 2.5 billion users monthly [6].

Regarding Jordan, it has been listed Facebook as the most popular, with $88.6 \%$ of all social networking users. When it comes to languages [7]. English has been classified as the top language with $25.3 \%$ of internet content, while Arabic content represents no more than 4.8\% [8], [9]. For English content published on Facebook, FTS allows Arabs to read the English posts in Arabic.

However, the number of Facebook active monthly users in the Middle East reaches 200 million [10]. A study conducted in Jordan to elicit information about the source of information for a sample of 500 medical students in three Jordanian Universities. They found that $83.4 \%$ used social media and $83.4 \%$ favored online search to access COVID-19 related information. However, the study did not specify the most popular social networks among medical Students [11].

Social media becomes an essential means of communication since COVID-19 updates take place around the clock. Such widespread use, particularly at the time of a health crisis mandating social isolation, raises questions about the content Facebook provides in terms of its effectiveness and limitations to end users since Arab Facebookers are mostly exposed to posts written in English. Some Arab Facebookers can read and understand English. However, there is still a large portion of users who are not sufficiently competent to read posts in English, and instead, use FTS, Facebook's translation service since 2011. Facebook Translate helps users communicate with each other in different languages. Facebookers can easily activate the translation service on their profiles. Users can select their region and language and the language they wish their posts to be translated into in their settings.

\section{Facebook Translation Service (FTS)}

Historically, Facebook initially used Google Translate service to translate the posts into 50 languages. Facebook launched its first Translation Service in 2011, called In-line Translation Facebook Tool [12], this time powered by Microsoft Bing. For example, if a non-Spanish speaker gets a cross a Spanish Facebook post, he will be able to see the translation of the post in his own language.

Currently, FTS offers translation services for 89 languages, and they will continue to add more languages. FTS adopted a phraseto- phrase MT approach by translating whole sequences of words, where the lengths may differ. In 2017, Mark Zuckerberg, the founder of Facebook, explained that translation is the best means to connect humans globally. Language understanding brings people close and thus help them to share and get access to information universally [13]. In the same year, the Facebook research team developed a new MT approach using Convolutional Neural Networks (CNNs) instead of Google Translate. The recurrent neural networks can help translate languages more accurately (increase quality on a BLEU scale) and up to nine times faster than the traditional recurrent neural networks (RNNs). Neural MT has been described approach as a tool to provide instant translation accurately across languages faster than previous MT approaches [14]. This is confirmed by [15], [16].They have shown that "CNNs have been very successful in several machine learning fields, such as image processing. However, recurrent neural networks (RNNs) are the incumbent technology for text applications and have been the top choice for language translation because of their high accuracy".

In late 2019, the Facebook research team announced new advances in NLP, boosting Facebook Translation accuracy. They have also introduced a new self-supervised pretraining approach, RoBERTa, that surpassed all existing Natural Language Understanding (NLU) systems on several language comprehension tasks. They have also collaborated with New York University (NYU), DeepMind Technologies, and the University of Washington (UW) to promote their future research [16].

The present study focusses on Facebook translation of English Facebook posts related to COVID-19 released by International Organizations such as WHO, political leaders, medical specialists, and the general public into Arabic. The study provides constructive feedback about whether FTS is considered a reliable source of information or not and the degree of trust among the end-users. It addresses the following questions:

What, according to participants, is the percentage of FTS Activation in Jordan?

What is the rate of using FTS in translating English Facebook posts and Covid-19 Facebook posts into Arabic?

As a source of information, how often Jordanians use FTS?

To what degree FTS output is adequate and fluent in English to Arabic translation?

To what extent, Jordanian rated FTS output?

\section{Literature Review}

The outbreak of COVID-19 makes social networking sites a source of information for many people around the globe. COVID19 is an emergent crisis, unprecedented in the size of information posted daily about the pandemic. Facebook usage increases by $40 \%$ due to the COVID-19 crisis. Facebook becomes a medium of communication. Machine Translation plays an influential role in facilitating communication among human communities [17], [8]. The study is mainly concerned with the popularity of FTS among Jordanians in translating the English Facebook posts into Arabic. To the best of knowledge of the author, this is the first research to fill the gap and provide the literature with information about the role of FTS in educating Jordanians and fighting COVID-19.

Social networking sites have become a useful tool in confronting the crisis and keeping people close to each other when they are isolated due to lockdown in most of global countries. He 
adds that the public health experts have used social networking sites to interact with the public and provide healthcare advice to fight and prevent the spread of the outbreak[[18]]. The acceleration in digital life changes how health information is approached. The health care providers used this opportunity to disseminate their advice and right information for the public [[19]].

On the other hand, Facebook becomes the source of information for 100,000 health care providers regarding the COVID-19 outbreak, "For the past few weeks, after I get home from my shifts in the emergency room, I have been scrolling through Facebook pages on my laptop, getting firsthand stories from doctors in Italy, China, and Iran" [20]. Another study has been conducted a descriptive study on medical students in Jordan to assess knowledge, attitude, perceptions, and precautionary measures toward COVID-19. They have found that $83.4 \%$ used social media sites as their preferred source of information regarding COVID-19 [11]. Moreover, another study has been carried out in Jordan to assess university students' knowledge, practice, and attitude regardless of their majors. They have found that university students' information source is social media, internet, and television. No significant difference was noticed between medical and non-medical college students on the sources of their information. On the other hand, social networking sites have become platforms for survival against social isolation during the COVID-19 [21]. In this regard, Facebook becomes the central platform to help the community and those in need during this pandemic [22].

Another use of social networks during the crisis is highlighted by as a platform to preach and provide spiritual support to conduct digital prays and biblical lesson [23]. On the other hand, social media shows how social networks become the platform for sharing controversial messages to Chinese people. Some users blamed China as a source of the disease and therefore Chinese became the target of many hateful and offensive posts on most social networking sites., such as Facebook and the like. Facebook and other social media have been clamped down the spread of fake news concerning COVID-19 [24], [25]. The study highlights the FTS infighting world crisis, COVID-19, in provide translation for the English Facebook posts related to COVID-19 into Arabic.

\section{Methodology}

Arabic is the official language of 24 Arab countries with a population of 300 million. Jordan is one of Arab countries and the research on Facebook Translation service in Jordan would of great interests and a source of information for all Arabs. Descriptive cross-sectional study design was used in this work. Our sample consisted of 200 Jordanians from five cities of Jordan: Ajlun, Amman, Irbid, Jerash, Kerak. The study population has different educational backgrounds except for English language specialists (Linguistics, literature, and Translation studies) and were asked to fill the questionnaire. The study used an online questionnaire delivered to participants in the period between March 10 to July 29 during the lockdown in the Jordanian government's effort to control the spread of COVID-19. The online questionnaire was created on Google Forms and sent to the participants through Facebook Messenger to ensure the social distancing in line with the Jordanian government's recommendations. Therefore, all participants in this study have Facebook pages. The study's total population was 200 responded to the study, 106 females (52.7\%) of the sample, and 95 male participants (47.3\%) (see appendix 1) An independent T-test was conducted to identify a relation between rural and urban areas regarding rating the adequacy and fluency of FTS. It is evident from the table 2 that a value of 1.076 at $\operatorname{sig}=0.283$ indicates that there are no statistically significant differences at 0.05 between rural and urban residents in the evaluation of the fluency of FTS. Moreover, another t-test was conducted to verify the relation between gender and fluency rating. It is evident from the table 3 that a value of $t$ was 1.145 at sig = 0.254 , indicating that there were no statistically significant differences at 0.05 between males and females in evaluating the fluency of Facebook Translation Service. Another also t-test conducted on the relation between the education level and the adequacy. The study found that there are statistically significant differences at 0.05 in evaluating the fluency of FTS according to the educational level, and by conducting post-tests to test the analysis of variance, it was found that the source of the differences is the existence of statistically significant differences between holders of a diploma and those who hold a master's degree in favor of a diploma.

\subsection{Research Instrument}

The researchers designed a dichotomous questionnaire that was created on the Google Forms platform. The questionnaire has yes/no questions to elicit Jordanians' responses concerning their usage of Facebook Translation service in translating English Facebook posts into Arabic and, in particular COVID-19 posts.

The online questionnaire consisted of seven main divisions main sections. The first section collected demographic data on gender, age, education, cities, and living areas. The second section consisted of the following questions:

Have you activated FTS on your Facebook Page?

Have you used FTS in translating English Facebook posts into Arabic?

Have you used FTS in translating English Covid-19 Facebook posts into Arabic?

Have you considered FTS output as a Primary source of your information regarding COVID-19?

Irrespective of the English posts' contents (maybe not reliable, fake, or misleading), do you find the translation by FTS acceptable?

Irrespective of the English posts' contents (maybe not reliable, fake, or misleading), do you think Facebook Translation Service has translated them accurately?

Have you rated FTS?

Two professors reviewed the survey, and proper modifications were completed before delivering to participants. Participation in the study was voluntary, and personal Facebook pages and identifiers were not collected. Data was imported into Excel for management and then SPSS for analysis. Numbers and percentages were presented for all variables. 


\section{Results}

\subsection{FTS activation among Jordanians}

First, we examined the number of Facebookers who activated FTS on their Facebook pages. As mentioned above, the questionnaire was sent via Facebook Messenger, and therefore all participants have Facebook pages. Our analysis showed that $(87.5 \%)$ of the participants activated FTS on their Facebook, while only $(12.5 \%)$ of the participants did not activate FTS. This finding shows that FTS is used mostly by Jordanian. Moreover, this result correlates with [[7]] that Facebook is the most popular social media.

Table 1: Percentage of FTS Activation

\begin{tabular}{lrr} 
Classification & Sum of Frequency & \multicolumn{3}{c}{ Sum of Percent \% } \\
\hline No & 25 & 12.5 \\
Yes & 175 & 87.5 \\
\hline Grand Total & $\mathbf{2 0 0}$ & $\mathbf{1 0 0}$
\end{tabular}

\subsection{Have you used FTS in rendering English Facebook posts into Arabic?}

Next, we wanted to know if Jordanians used FTS in rendering English Facebook posts into Arabic in general and English posts concerning COVID-19 rendering into Arabic. Our analysis showed that $(87.6 \%)$ of Jordanians used FTS in rendering English posts into Arabic, while $12.4 \%$ did not use FTS in translation. These results highlight the role of FTS in facilitating communications between English and Arabic.

Table 2: FTS usage among Jordanians at the time of Covid-19

\begin{tabular}{lrlr}
$\begin{array}{l}\text { Have you used Facebook Translation Service in rendering English } \\
\text { posts into Arabic? }\end{array}$ & $\begin{array}{l}\text { Sum of Percent } \\
\text { \% }\end{array}$ \\
\hline Classification & Sum of Number of respondents & \\
\hline No & 25 & 12.4 \\
Yes & 175 & 87.6 \\
\hline Grand Total & $\mathbf{2 0 0}$ & $\mathbf{1 0 0}$ \\
\hline
\end{tabular}

\subsection{Have you used FTS in translating English Facebook Covid- 19 posts into Arabic?}

We aimed to elicit information about the percentage of FTS in translating English Covid-19 posts into Arabic. The study's analysis showed that (83.1\%) have used FTS in translating English Facebook COVID-19 posts into Arabic. These figures showed that FTS played a significant role in facilitating communications among Jordanians at the time of the COVID-19 outbreak in Jordan.

Table 3: FTS rendering English posts about Corona Virus (COVID-19) into Arabic

Have you used Facebook Translation Service in rendering English posts about Corona Virus (COVID-19) into Arabic?

Classification

Sum of Frequency

Sum of Percent $\%$

\begin{tabular}{lcc} 
No & 33 & 16.9 \\
Yes & 167 & 83.1 \\
\hline Grand Total & $\mathbf{2 0 0}$ & $\mathbf{1 0 0}$
\end{tabular}

5.4. Have you used FTS daily at the time of COVID-19?

Next, we aimed to identify how often Jordanians used FTS during the time of lockdown in Jordan. Our analysis showed that most of Jordanians used FTS daily with (94. \%). This correlates with [[7]] indicated that Facebook usage in Jordan reached 92\% over the COVID-19 period, while only (5.97\%) did not use it daily.

Table 4: Frequency Facebook and FTS among Jordanians at the time of Covid19 lockdown

\begin{tabular}{lrr} 
Classification & Sum of Frequency & Sum of Percent \% \\
\hline Daily & 188 & 94 \\
Rarely & 1 & 0.5 \\
Weekly & 11 & 5.5 \\
\hline Grand Total & $\mathbf{2 0 0}$ & $\mathbf{1 0 0}$
\end{tabular}

\subsection{Have you considered FTS output as a Primary source of your information regarding COVID-19?}

First, we examined how the participant deals with FTS as a source of information. The question aimed to identify whether Jordanians considered FTS output as sources of information at the time of lockdown. Our analysis showed that most participants with $(62.2 \%)$ considered FTS output as the primary source of their information regarding COVID-19. In comparison (27.8\%) showed that they considered FTS as a secondary source of their information.

Table 5: Facebook as a Sources of Information Related to COVID-19

\begin{tabular}{lrr}
\hline Category & Sum of Frequency & Sum of Percent \% \\
\hline Partial & 3 & 1.5 \\
Minor & 16 & 8.5 \\
Primary & 125 & 62.2 \\
Secondary & 56 & 27.8 \\
\hline Grand Total & $\mathbf{2 0 0}$ & $\mathbf{1 0 0}$ \\
\hline
\end{tabular}

5.6. How do you rate the fluency of FTS output?

The above chart illustrates how the study's participants rated FTS. The analysis showed that the respondents rated FTS output at the category of almost all of the meaning in the translation with $41 \%$, followed by $40.5 \%$ who believed that FTS output is complete as fluent. However, $14 \%$ of the responses showed that the translation is not fluent, and FTS output contains some of the ST meaning, followed by $4.5 \%$ for FTS as disfluent.

\section{Table 6: FTS Adequacy}

\begin{tabular}{lrr}
$\begin{array}{l}\text { Adequacy } \\
\text { All the meaning in the source is } \\
\text { contained in the translation, no } \\
\text { more, no less. }\end{array}$ & $\begin{array}{l}\text { Sum of } \\
\text { Frequency }\end{array}$ & $\begin{array}{l}\text { Sum of } \\
\text { Percentage }\end{array}$ \\
$\begin{array}{l}\text { Almost all the meaning in the source } \\
\text { is contained in the translation. }\end{array}$ & 81 & 40.5 \\
$\begin{array}{l}\text { Fragments of the meaning in the } \\
\text { source are contained in the } \\
\text { translation. }\end{array}$ & 82 & 41 \\
$\begin{array}{l}\text { None of the meaning in the source is } \\
\text { contained in the translation. }\end{array}$ & 28 & 14 \\
$\begin{array}{l}\text { Grand Total } \\
\text { Grand }\end{array}$ & 9 & 4.5 \\
\hline
\end{tabular}




\subsection{Have you rated the adequacy of FTS output?}

The above chart elucidates how the study respondents rated FTS output fluency (grammar). It showed that the majority of respondents rated FTS output as fluent with minor errors at the percentage of $69.65 \%$, followed by $23 \%$ indicated that FTS output rated FTS output as fluent with no errors. On the other hand, $6.5 \%$ rated FTS output as disfluent.

Table 7: FTS Fluency

\begin{tabular}{lrr} 
Fluency & $\begin{array}{l}\text { Sum of } \\
\text { Frequency }\end{array}$ & $\begin{array}{l}\text { Sum of } \\
\text { Percentage }\end{array}$ \\
\hline $\begin{array}{l}\text { Refers to a perfectly flowing text with no } \\
\text { errors. }\end{array}$ & 46 & 23 \\
$\begin{array}{l}\text { Refers to a smoothly flowing text even } \\
\text { when a number of minor errors are }\end{array}$ & 139 & 69.5 \\
present. & 13 & 6.5 \\
$\begin{array}{l}\text { Refers to a text that is poorly written and } \\
\text { difficult to understand. }\end{array}$ & 2 & 1 \\
$\begin{array}{l}\text { Refers to a very poorly written text that is } \\
\text { impossible to understand. }\end{array}$ & $\mathbf{2 0 0}$ & $\mathbf{1 0 0}$ \\
\hline Grand Total & &
\end{tabular}

\subsection{Have you rated FTS output?}

Then, the study aimed to elicit information about the FTS output rating by the participants. The analysis showed that $55.5 \%$ of respondents rated FTS sometimes, $42 \%$ rated FTS always, while $2.5 \%$ were not sure they rated FTS. The study here emphasizes that rating is crucial to improve its service and provide the end-users with information about the end users' trust of FTS output development of FTS service.

Table 8: FTS Rating

\begin{tabular}{lrrr} 
& \multicolumn{3}{l}{ Sum of Percent } \\
Classification & Sum of Number of respondents & \multicolumn{2}{c}{$\%$} \\
\hline Always & 84 & 42 \\
Not sure & 5 & 2.5 \\
Sometimes & 111 & 55.5 \\
\hline Grand Total & $\mathbf{2 0 0}$ & $\mathbf{1 0 0}$
\end{tabular}

\section{Discussion}

The current descriptive study assessed the popularity of FTS in translating English Facebook posts into Arabic and Posts related to COVID-19 in particular. Participants were found to be familiar with FTS usage during the COVID-19 lockdown. The results showed that social networking sites have a significant role in our modern life. Facebook becomes one of the most popular social media websites. Facebook becomes a source of information during the time of COVID-19 lockdown since Facebookers read posts around the clock. The study showed that (94\%) of participants used Facebook daily to read, share, and post. This percentage complied with [[7]] that Facebook occupied the first rank in social media stats with (88.69\%) over June 2020 in Jordan.

Our assessment of the information sources showed (62.2\%) of Jordanians considered FTS as the primary source of their information during the COVID-19 lockdown. The study attunes with what [[11]] and [[21]] said in their research that Facebook becomes the source of information for medical students in Jordan (83.4\%). This means that Facebook becomes a popular online platform that supports the efforts to fight COVID-19 at the government side, where the government officials and health care providers to convey their messages to control the outbreak of COVID-19. Even though FTS became a source of information in translating English Facebook posts into Arabic, but the need for a thorough analysis of the output of FTS by a translation scholar is of great importance to highlight the limitations and strengths of FTS.

The study indicated that FTS was used daily, equal to (94\%). Moreover, it is found that Jordanians used FTS in translating COVID-19 posts into Arabic, with a percentage of (83.1\%). This finding complied with [[11]] finding that $(83.4 \%)$ medical students in Jordan used Social Media to learn about COVID-19. However, they did not ask the participants about their preferred social media platform. This finding motivates the researcher to conduct further study to verify the capacity of FTS to provide adequate and fluent output to the end-users.

The study found that the participants rated FTS output adequacy with (41\%) almost the meaning of the ST in the TL; (40.5\%) showed that FTS output is fully adequate to the end-user. Furthermore, it is found that (69.65) of FTS output has minor errors, while (23\%) is free of errors. On the other hand, the study highlighted that Jordanians sometimes tended to rate FTS service with (55.5\%) for rating FTS and always rating for FTS with (42\%). The rating provides a review for the FTS team to improve its services. The rating benefits Facebook in two ways: help the users make decisions to use FTS or not and help Facebook gain the users' credibility.

\section{Conclusion}

Overall, Facebookers in Jordan tend to use FTS in rendering English posts in general and posts related to COVID-19 into Arabic. This means that Facebookers used Facebook as their primary source of information. The study concludes that in such an unprecedented COVID-19 crisis, the health officials should create Facebook Profiles with a blue tick for medical information during crises. Countries where the outbreak is hitting hard should focus on educating the public. Moreover, medical specialists and translation scholars should form evaluation groups to ensure that FTS can render COVID-19 medical posts into Arabic fluently and adequately. Finally, the study recommends further studies to scrutinize FTS outputs thoroughly to highlight the service's limitations and strengths.

\section{Conflict of Interest}

The authors declare no conflict of interest.

\section{Acknowledgment}

The authors would like to extend their thanks to all participants in this study. The research has been funded by Applied Science Private University, Jordan.

\section{References}

[1] Z. Almahasees, Diachronic Evaluation of Google Translate, Microsoft Translator and Sakhr in English-Arabic Translation - the UWA Profiles and Research Repository, 2020.

[2] Jack, The Top 3 Translation Industry Trends for 2019, 2019.

[3] Z.M. Almahasees, "Assessment of Google and Microsoft Bing Translation of Journalistic Texts," International Journal of Languages, Literature and Linguistics, 2018, doi:10.18178/ij111.2018.4.3.178. 
[4] Omniscen Technologies, How fast is machine translation? : Language Studio Help Desk, 2016.

[5] KH, How Big Data And Machine Translation Combine To Fight COVID-19, 2020.

[6] Kenton, Social Networking Definition, 2020.

[7] Statcounter, Social Media Stats Jordan | StatCounter Global Stats, 2020.

[8] Z.M. Almahasees, "Assessing the Translation of Google and Microsoft Bing in Translating Political Texts from Arabic into English," International Journal of Languages, Literature and Linguistics, 2017, doi:10.18178/ij1ll.2017.3.1.100.

[9] Sistanis, Top 10 Languages Used On the Internet for 2020 - Speakt.com, 2020.

[10] Debusmann, Facebook teams up with Middle East firm to combat Covid-19 rumours - Arabianbusiness, 2020.

[11] A.I. Khasawneh, A.A. Humeidan, J.W. Alsulaiman, S. Bloukh, M. Ramadan, T.N. Al-Shatanawi, H.H. Awad, W.Y. Hijazi, K.R. Al-Kammash, N. Obeidat, T. Saleh, K.A. Kheirallah, "Medical Students and COVID-19: Knowledge, Attitudes, and Precautionary Measures. A Descriptive Study From Jordan," Frontiers in Public Health, 2020, doi:10.3389/fpubh.2020.00253.

[12] Singh, How to enable new Facebook In-Line Translation Tool on Facebook Pages | Tech 24 Hours, 2020.

[13] Knowledge Complexity, A recipe for intimacy?, 2017.

[14] J. Gehring, M. Auli, D. Grangier, D. Yarats, Y.N. Dauphin, "Convolutional sequence to sequence learning," in 34th International Conference on Machine Learning, ICML 2017, 2017.

[15] Facebook AI, New research awards in NLP and machine translation, 2019.

[16] Facebook Engineering, A novel approach to neural machine translation Facebook Engineering, 2017.

[17] Z. Almahasees, "Machine Translation Quality of Khalil Gibran's The Prophet," Arab World English Journal For Translation and Literary Studies, 2017, doi:10.24093/awejtls/vol1no4.12.

[18] T. Loren, Social Media \& Covid-19: How to Communicate During a Crisis - Later Blog, 2017.

[19] Garza, How Social Media Is Shaping Our Fears of the Coronavirus | Time, 2020.

[20] Zakrzewski, The Technology 202: Coronavirus could change how social networks approach public health - The Washington Post, 2020.

[21] H. Alzoubi, N. Alnawaiseh, A. Al-Mnayyis, M. Abu-Lubad, A. Aqel, H. AlShagahin, "Covid-19 - Knowledge, attitude and practice among medical and non-medical university students in Jordan," Journal of Pure and Applied Microbiology, 2020, doi:10.22207/JPAM.14.1.04.

[22] H. Ouyang, "At the Front Lines of Coronavirus, Turning to Social Media," The New York Times, 2020.

[23] Heilweil, Coronavirus social distancing leads to empty churches and a rise in apps - Vox, 2020.

[24] Wong, Coronavirus sparks a different kind of problem for social networks CNET, 2020.

[25] Scott, Facebook to tell millions of users they've seen 'fake news' about coronavirus - POLITICO, 2020.

\section{Appendices:}

Appendix 1: Frequency and Percent for demographic variable

\begin{tabular}{|c|c|c|c|c|}
\hline Variable & \multicolumn{2}{|c|}{ Classification } & Frequency & Percent \% \\
\hline \multirow{2}{*}{ Gender } & \multirow{2}{*}{\multicolumn{2}{|c|}{ Male }} & 95 & 47.3 \\
\hline & & & 105 & 68.2 \\
\hline \multirow{5}{*}{ Education } & \multicolumn{2}{|c|}{ Bachelor } & 46 & 22.9 \\
\hline & \multicolumn{2}{|c|}{ Diploma } & 87 & 43.3 \\
\hline & \multicolumn{2}{|c|}{ High School } & 10 & 5.0 \\
\hline & \multicolumn{2}{|c|}{ Master } & 35 & 17.4 \\
\hline & \multicolumn{2}{|c|}{$\mathrm{PhD}$} & 21 & 10.4 \\
\hline \multirow{10}{*}{ Cities } & \multirow[t]{2}{*}{ Amman } & Urban & 50 & $25 \%$ \\
\hline & & Rural & 0 & 0 \\
\hline & \multirow[t]{2}{*}{ Ajlun } & Urban & 19 & 9.5 \\
\hline & & Rural & 11 & 5.5 \\
\hline & \multirow[t]{2}{*}{ Jerash } & Urban & 26 & 13 \\
\hline & & Rural & 9 & 4 \\
\hline & \multirow[t]{2}{*}{ Irbid } & Urban & 30 & 15 \\
\hline & & Rural & 20 & 10 \\
\hline & \multirow[t]{2}{*}{ Kerak } & Urban & 21 & 10.5 \\
\hline & & Rural & 15 & 7.5 \\
\hline & \multicolumn{2}{|l|}{ Total } & 200 & 100 \\
\hline
\end{tabular}

